\title{
PHYSICALLY MEANINGFUL DICTIONARIES FOR EO CROWDSOURCING: A ML FOR BLOCKCHAIN ARCHITECTURE
}

Mihai Coca, Iulia Neagoe and Mihai Datcu

\section{ABSTRACT}

Due to the complexity and difficult comprehension of remote sensing data, ubiquitous methods for content exploitation are difficult to identify. The main limitation is ascribed to the fact that different perceptions, individual human experts and non-identical remote sensors fabricate a total heterogeneous context. In order to overpass these breaches, an interactive learning model is demanded, where different experts, participating into a validation process, expose and verify shared dictionaries based on users' understanding. We propose a federated system for collaborative labeling of remote sensing datasets that transform blockchain storage into blockchain knowledge. This writing wants to provide insights into a decentralized methodology, capable of building a publicly available, largescale benchmark data for image classification and hosting a permanently updated machine learning (ML) model.

Index Terms- Earth observation (EO) data, machine learning, blockchain technology, Inter-Planetary File System (IPFS) 